\section{Effect of Oatmeal on Postprandial Vascular Compliance Following a High Fat Meal}

\section{Abstract}

Background and aim: Postprandial hyperlipidaemia has been associated with acute cardiovascular effects e.g. endothelial dysfunction and peripheral vasodilatation. Oats have known health benefits, and may reduce the transient, post-prandial endothelial dysfunction. The aim of this short study was to investigate if markers of endothelial function pulse wave velocity (PWV) and augmentation index corrected for heart rate (Alx@75) are affected by a meal with varying levels of saturated fat, with or without oatmeal.

Methods and results: Fourteen subjects (aged between 22 and 51 years) were recruited. On their first visit, following baseline measurements of anthropometry, blood pressure (BP), heart rate (HR), PWV and Alx@75, they were given one of three meals (low fat, high fat, and high fat with 60g oatmeal) in a randomised order. Subjects returned three hours later for re-assessment of BP, PWV and Alx@75. Visits two and three followed the same protocol, but only BP, HR, PWV and Alx@75 were measured. There was a significant correlation between baseline PWV and systolic pressure $(p<0.02)$, and Alx@75 and diastolic pressure $(p<0.005)$. Alx@75 was positively related to BMI and waist circumference $(p<0.01$ and $p<0.005$ respectively). Alx@75 fell following the high fat meal $(p<0.05)$, but not when oats were taken. Heart rate increased following the high fat meal with oats $(p<0.05)$, but no differences were found between fasting and postprandial PWV after any of the meals.

Conclusion: Alx@75 was correlated with BMI and waist circumference. The decrease in Alx@75 following the high fat meal requires further investigation, and Alx@75 is possibly a more sensitive marker of arterial compliance than PWV in a young healthy population.

Keywords: Fat diet; Oatmeal; Postprandial vascular compliance; Pulse wave velocity; Augmentation index

\section{Devlin N, McKenzie J and Gow IF}

Queen Margaret University, Musselburgh, Scotland

\section{Corresponding author: lain F Gow}

Dietetics, Nutrition, and Biological Sciences, Queen Margaret University, Queen Margaret University Drive, Musselburgh, EH21 6UU, Scotland.

झ igow@qmu.ac.uk

Tel: +44 1314740000

Citation: Devlin N, McKenzie J, Gow IF. Effect of Oatmeal on Postprandial Vascular Compliance Following a High Fat Meal. J Clin Nutr Diet. 2016, 2:1.

Received: September 08, 2015; Accepted: February 03, 2016; Published: February 12,2016

\section{Introduction}

Cardiovascular disease (CVD) is the leading cause of death in the adult population worldwide, with over 7 million related deaths annually. Known risk factors contributing to the development of CVD are obesity, sedentary behaviour, poor lipid profiles, hypertension and diabetes [1]; non-modifiable risk factors include age, gender and family history [1], but these are less important in terms of promoting overall health. Changes in the structure and responsiveness of the blood vessel wall brought about by atherosclerosis are possibly the most important underlying pathological process for cardiovascular disease [2]. A change in endothelial function is one of the earliest warning signs of cardiovascular disease, which occurs naturally with age [3].

Currently non-invasive methods such as flow mediated dilatation (FMD), pulse wave velocity (PWV) and pulse wave analysis (PWA) are used identify the risk of CVD, e.g. endothelial dysfunction, arterial compliance, central systolic pressure [4]. PWV has emerged as an independent predictor of CVD prognosis [5], and has significant relationships with body composition, blood pressure, dietary and lifestyle factors. Augmentation index (Alx) is one form of pulse wave analysis (PWA) and is determined by 
arterial compliance and peripheral wave reflections [6]. Since Alx decreases with increasing heart rate $[7,8]$, Alx should be corrected to a value relative to a heart rate of 75 bpm, Alx@75 $[7,8]$.

Obesity, especially central obesity is an important risk factor for cardiovascular disease, and around $25 \%$ of men and $26 \%$ of women are classified as being obese in Scotland [9]. In addition, the percentage of men with a raised waist circumference $(>102$ $\mathrm{cm}$ ) reflecting visceral and central adiposity, is steadily rising, with $25 \%$ of men and $34 \%$ of women $(>88 \mathrm{~cm}$ ) being at increased risk.

Recommendations for dietary fat intake in the UK are currently $<35 \%$ of total food energy, as $<11 \%$ saturated fat, $6.5 \%$ polyunsaturated fat, $13 \%$ mono-unsaturated and $<2 \%$ trans fats [10]. A single high saturated fat meal negatively affects endothelial function, as measured by FMD [11-13] and PWV [14, 15] with additional increases in blood pressure and triglyceride concentrations [16], although Djousse et al. [17] found no significant effects of a high fat meal on FMD or PWV respectively.

Vogel et al. [11] further explored the acute effects of fat on cardiovascular health, by testing meals which included extra virgin olive oil, canola oil and canned red salmon (the latter two meals containing 5-6 g of omega-3 fatty acids). Results clearly indicated deterioration in FMD (interpreted as impairment of endothelial function) following the olive oil-based meal as compared with the omega-3 rich meals, suggesting saturated fat may not be the only type of fat to negatively affect cardiovascular health. Raitakari et al. [18] also investigated the effects of different fats, limiting the study to saturated and mono-unsaturated fats. Like Vogel et al. [11], findings showed a significant reduction in FMD following the high mono-unsaturated fat meal, thus these studies suggest a beneficial effect of omega- 3 fatty acids on endothelial function with poor support for unsaturated and mono-unsaturated fats.

Studies with oats have indicated that this cereal could influence blood glucose and cholesterol levels [19], an effect which does not seem to exist with other cereals [20] and prompting health campaigns to promote oats as having the ability to reduce the risk of heart disease (e.g. 'The Heart Heath Roadshow' [21]). Much of the research has concentrated on the ability of oats to improve lipid profiles, to lower blood pressure and to control blood glucose; positive results have primarily emerged promoting the addition of oats to the daily diet [22]. Among others, the $\beta$-glucan content is considered to be an active component of the cholesterol lowering effect [23]. While the effects of $\beta$-glucan and other soluble fibres have been associated with reduced risk of CVD, their effect on endothelial function has had limited investigation. Katz et al. [24] found that oats prevented a decrease in endothelial responses after a high fat meal whereas wheat intake failed to have an effect. In a follow-on study, Katz et al. [25] failed to find significant benefits of acute or long term oat intake on endothelial function, as measured by FMD, although the trends pointed to an improvement. The acute effects of a high fat meal on vascular function are still a debatable topic, with very inconsistent results, and hence this present study was aimed at assessing the effect of a single meal with low, or high fat content on endothelial function, and the effect of oats on the response to the high fat meal. The aim of this study therefore was to investigate if a meal rich in saturated fat with or without oats affects arterial compliance, as measured by pulse wave velocity (PWV) and augmentation index corrected for heart rate (Alx@75).

\section{Methods}

This project had ethical approval from the Queen Margaret University Research Ethics Committee. A total of 14 subjects aged between 22 and 51 years volunteered to participate in the study. Subjects were recruited through Queen Margaret University (QMU) by a moderator email. All subjects volunteered to participate after being fully informed about the research. Each subject provided written consent in accordance with QMU's Research Ethics Committee. Subjects were excluded if they had previously been diagnosed with cardiovascular disease or type 2 diabetes (T2DM).

\section{Overall experimental design}

Subjects were required to make three visits to the testing lab at QMU. On the first visit, subjects were asked to complete a general health questionnaire and had a number of anthropometric measurements taken, i.e., height, body mass and waist circumference. Blood pressure (BP), heart rate (HR), pulse wave velocity (PWV) and augmentation index (Alx) were also measured. Subjects were required to fast overnight for 12 hours and to refrain from smoking and caffeine intake during this period. It was also requested that no strenuous physical activity to be performed in the 24 hours prior to the testing. After all measurements were complete, subjects were given one of three meals in random order and asked to consume $100 \%$ of the meal. Three hours after consumption of the meal, subjects were asked to return to the testing lab for blood pressure, heart rate, PWV and Alx@75 measures to be re-taken. During the three hour time interval subjects were asked to refrain from food and fluid intake (with the exception of water), smoking and exercise. Visits 2 and 3 followed the same protocol, but only blood pressure, heart rate, PWV and Alx@75 measures were taken.

\section{Meal composition}

One of the following three meals was provided on each of the three visits after fasting measurements were obtained. The meals (Table 1) were provided in a randomised order and subjects were asked to consume $100 \%$ of each meal.

\section{Meal A}

Meal A represented a low fat meal. In line with recommendations, it had $33 \%$ of energy coming from fat [10]. The meal consisted of: a white bread cheese sandwich (two slices of low fat cheddar cheese, $7 \mathrm{~g}$ low fat spread), $125 \mathrm{~g}$ low fat cheesecake, $200 \mathrm{ml}$ semi-skimmed milk.

\section{Meal B}

Meal B represented a high saturated fat meal and consisted of: a white bread cheese sandwich (two slices of cheddar cheese, $7 \mathrm{~g}$ butter portion), $125 \mathrm{~g}$ chocolate cheesecake, $200 \mathrm{ml}$ full fat milk.

\section{Meal C}

Meal $\mathrm{C}$ was a combination of Meal $\mathrm{B}+60 \mathrm{~g}$ of rolled oats served as porridge. The porridge was made using water, to which the subjects could add their $200 \mathrm{ml}$ of full fat milk if they wished. 
Table 1 Composition of the meals.

\begin{tabular}{|c|c|c|c|c|c|c|c|}
\hline Meal & & Fat (g) & Saturated Fat (g) & Fat as \% of Energy & Saturated Fat as \% of Energy & kCalories & Oats (g) \\
\hline A) & Low Fat & 26 & 13 & $33 \%$ & $16 \%$ & 700 & -- \\
\hline B) & High Fat & 60 & 37 & $57 \%$ & $35 \%$ & 950 & -- \\
\hline C) & High fat+oatcakes & 65 & 37 & $50 \%$ & $28 \%$ & 1160 & 60 \\
\hline
\end{tabular}

The $60 \mathrm{~g}$ serving of porridge also contained an estimated $3 \mathrm{~g}$ of $\beta$-glucan. There was a washout period of one week between each meal day $[24,26]$.

\section{Anthropometric measurements}

Height and body mass were measured to the nearest $0.1 \mathrm{~cm}$ and $0.1 \mathrm{~kg}$ respectively. Subjects were weighed bare foot and with excess clothing removed. BMI was calculated using the formula: (Weight $(\mathrm{kg}) /(\text { Height }(\mathrm{m}))^{2}$.

\section{Waist circumference}

Waist circumference was measured twice, with the subject in a standing position, to the nearest $0.1 \mathrm{~cm}$, with a non-elastic tape and an average taken. If the measurements differed by more than $2 \mathrm{~cm}$, a third reading was taken and the results averaged. The measurement was taken at the level of the trunk where the girth was minimal, or if there was no noticeable indentation, the measurement was taken midway between the lowest rib (laterally) and the iliocristale landmark.

\section{Blood pressure}

Resting blood pressure was taken in the morning of the three visits, and three hours after consumption of the test meal. An automated sphygmomanometer (Omron 705CP-II, UK) was used to measure blood pressure. The subject was asked to sit upright for at least 10 minutes prior to the measurement being taken. The cuff was then wrapped firmly around the upper arm at heart level, ensuring no kinks in the air hose. The artery marker on the cuff was aligned with the brachial artery. An appropriate cuff size was used to ensure accurate measurement. Three measurements were taken with a minimum of 1 minute apart. The first measurement was discarded and an average of the final two calculated, based on the procedures recommended by the UK Government and the American Hypertension Association [27, 28].

\section{Pulse wave velocity (PWV)}

PWV was measured using the Vicorder (Skidmore Medical, UK). The distance travelled by the pulse wave $(\mathrm{cm})$ was measured from the supra-sternal notch to the top of the femoral cuff. Subjects were asked to lie in a supine position with their head/chest at a 30 degree angle to the horizontal as per the manufacturer's instructions. A neck cuff was placed around the subjects' neck with the pressure pad over the right carotid area. A $100 \mathrm{~mm}$ wide blood pressure cuff was placed around the subjects' right upper thigh to measure the femoral pulse. Subjects were asked not to sleep nor speak during the measurements. The cuffs were each inflated to $60 \mathrm{~mm} \mathrm{Hg}$ and waveforms recorded. Three measurements were taken with a minimum of 1 minute between each measurement and the results averaged. This protocol was completed first in a fasted state (baseline) and three hours after each meal.

\section{Augmentation index (Alx)}

Augmentation Index (AIx) was also measured using the Vicorder. A $100 \mathrm{~mm}$ cuff was placed around the subjects' right upper arm. With the previously recorded brachial blood pressure as the calibrating measure, brachial arterial pressure waveforms were captured. For a single reading the waveforms were collected for a minimum of 12 seconds or until a steady pattern of waves were established. Three measurements were taken with a minimum of 1 minute between each measurement, and the results averaged. Alx@75 was assessed in a fasted state and 3 hours after each meal.

\section{Statistical analysis}

Graphpad Prism (v5.01, Graphpad Software, USA) was used to analyse and plot the data, with statistical significance being accepted at the $p<0.05$ level of confidence. One way or two way analysis of variance (1- or 2- way ANOVA, with Bonferroni correction for multiple testing) and Student's paired "t test" were used to test for significance amongst variables, or between paired observations. Comparisons were by standard linear regression. The independent variables were Systolic BP, Diastolic BP, Body Mass Index, and Waist Circumference; the dependent variables were Pulse Wave Velocity and Augmentation Index corrected for heart rate at $75 \mathrm{bpm}$. Results are shown as mean values \pm SEM

\section{Results}

\section{Subject characteristics}

The physical characteristics for the entire study population are shown in Table 2. A total number of 14 subjects were involved in the study, and all subjects completed each stage of the research. Subjects were aged between 22 and 51 years and had no previous diagnosis of CVD or diabetes.

\section{Baseline relationships}

These are summarised in Table 3. PWV was correlated with systolic but not diastolic blood pressure $(p<0.02, r=0.618)$, whereas Alx@75 was not related to systolic, but showed a positive, linear relationship with diastolic pressure $(p<0.005$, $r=0.741$ ). There were no significant correlations between either waist circumference or $\mathrm{BMI}$ and $\mathrm{PWV}$, but both $\mathrm{BMI}$ and waist circumference showed a significant positive relationship with Alx@75 ( $p<0.01, r=0.682$ and $p<0.005, r=0.719$ respectively).

\section{Effects of meals}

Baseline and postprandial measures of blood pressure, PWV and Alx@75 measures are presented in Table 4 for each of the interventions employed in the study. There were no significant differences amongst the baseline values for each parameter with respect to meal type prior to each test meal, nor amongst the magnitude of post-prandial response (1 and 2-way ANOVAs). 
None of the meals produced any changes in PWV. Heart rate increased significantly ( $+4.4 \mathrm{bpm} 95 \% \mathrm{Cl} 0.3$ to $8.5 \mathrm{bpm}$ ) following the high-fat meal with oats $(\mathrm{p}<0.05)$, and Alx@75 decreased $(-3.9 \%, 95 \% \mathrm{Cl}-7.6$ to $-0.2 \%)$ after the high-fat meal $(p<0.05)$, an effect which was abolished when oats were incorporated in the high-fat meal.

\section{Discussion}

The influence of a high-fat meal, body mass index (BMI) and waist circumference (WC) on the surrogate markers of endothelial function pulse wave velocity (PWV) and augmentation index (Alx) were the key areas of interest. As expected, this study found positive correlations between Alx, BMI and WC, but no associations with PWV were found which is in agreement with previous studies investigating the relationship between Alx, BMI and WC which have found these measures of adiposity to be strong predictors of arterial compliance [29].

The major aim of this study was to investigate the acute effects of a high fat meal on arterial compliance, and the effect of oats. It was hypothesised that a high fat meal would decrease arterial

Table 2 Physical characteristics of study sample.

\begin{tabular}{|l|c|c|}
\hline Physical Characteristics & Mean Value & Total N \\
\hline Age $(\mathrm{yrs})$ & $31.0 \pm 2.1$ & 14 \\
\hline Body Mass $(\mathrm{kg})$ & $72.7 \pm 4.2$ & 14 \\
\hline Height $(\mathrm{cm})$ & $170 \pm 2$ & 14 \\
\hline Body Mass Index $\left(\mathrm{kg} \cdot \mathrm{m}^{-2}\right)$ & $24.1 \pm 1.1$ & 14 \\
\hline Waist Circumference $(\mathrm{cm})$ & $78.7 \pm 3.3$ & 14 \\
\hline
\end{tabular}

Table 3 Correlations between baseline (pre meal) parameters.

\begin{tabular}{|l|c|c|c|c|c|} 
& \multicolumn{2}{|c|}{$\begin{array}{c}\text { Pulse Wave } \\
\text { Velocity }\left(\mathrm{m} \cdot \mathrm{s}^{-1}\right)\end{array}$} & $\begin{array}{c}\text { Augmentation } \\
\text { Index at } 75 \mathrm{bpm} \\
(\%)\end{array}$ & \\
\hline Baseline Parameter & $\mathbf{r}$ & $\mathbf{p}$ & $\mathbf{r}$ & $\mathbf{p}$ & Total N \\
\hline Systolic BP $(\mathrm{mm} \mathrm{Hg})$ & 0.618 & $<0.02$ & 0 & NS & 14 \\
\hline Diastolic BP $(\mathrm{mm} \mathrm{Hg})$ & 0.432 & NS & 0.741 & $<0.005$ & 14 \\
\hline Body Mass Index $\left(\mathrm{kg} \cdot \mathrm{m}^{-2}\right)$ & 0.286 & NS & 0.682 & $<0.01$ & 14 \\
\hline Waist Circumference $(\mathrm{cm})$ & 0.421 & NS & 0.719 & $<0.05$ & 14 \\
\hline
\end{tabular}

compliance as previously reported [14, 15]. Instead our finding supported that of Berry et al. [30], in that a high fat meal has no acute effects on arterial compliance as measured by PWV. Previous studies have produced conflicting results, and although the type of fat has proven to be an important factor, our chosen high fat meal contained a significant proportion of saturated fat (35\% of total energy), which has previously been shown to negatively affect arterial compliance [31]. In addition this study adhered to the guidelines advised by [32], on how best to prepare subjects for PWV measurement, i.e., fasting, avoidance of caffeine and smoking, positioning of subject during the measurement. However, the significant decline in Alx@75 (also a measure of arterial compliance) following the high fat meal confounds matters further. Assuming that Alx@75 is an indicator of arterial compliance, this implies that the consumption of a high fat meal, improves arterial compliance. Although this is contrary to what was hypothesised by us, Westerbacka et al. [33] also found similar results. The fact that Alx@75 decreased significantly following the high fat meal suggests that the fat component may still have a role to play. Alternatively, it may be due to the higher calorie content of the high fat versus the low fat meal. This is a limitation of our study, whereby the high fat meal contained $36 \%$ more calories than the low fat control. However the most plausible mechanism for our finding is likely to be related to the action of insulin, although the hormone was not measured in this study. Insulin bound to its receptor has been found to have a vasodilatory effect through the release of nitric oxide (NO), by increasing endothelial nitric oxide synthase (NOS [34]). Raitakari et al. [18] found a direct relationship between forearm blood flow and the rise in insulin levels following and reported a significant increase in baseline diameter using FMD, though the apparent reduction in FMD\% itself did not reach statistical significance. A similar change in baseline arterial diameter has been reported by Harris et al. [35] following a high-fat meal. In addition, the inhibition of eNOS has been found to increase aortic compliance, as measured by PWV [36]. This would suggest that if the vasodilatory effects of insulin occurred in this study, due to increased activity of eNOS, markers of arterial compliance would not be negatively affected by the high fat meal, and indeed may explain the improvement observed here since the arteries are already in a dilated state. Westerbacka et al. [33] further

Table 4 Effect of a low fat, high fat, or high fat meal with oats on cardiovascular characteristics.

\begin{tabular}{|c|c|c|c|c|c|c|c|c|c|c|}
\hline \multirow{3}{*}{$\begin{array}{l}\text { Cardiovascular } \\
\text { Characteristics }\end{array}$} & \multicolumn{9}{|c|}{ Meal } & \multirow{3}{*}{$\mathbf{N}=$} \\
\hline & \multicolumn{3}{|c|}{ Low Fat } & \multicolumn{3}{|c|}{ High Fat } & \multicolumn{3}{|c|}{ High Fat + Oats } & \\
\hline & Pre & Post & $\begin{array}{l}\text { Change } \\
\text { (LCl-UCl) }\end{array}$ & Pre & Post & $\begin{array}{l}\text { Change } \\
\text { (LCl-UCI) }\end{array}$ & Pre & Post & $\begin{array}{l}\text { Change } \\
\text { (LCI - UCI) }\end{array}$ & \\
\hline $\begin{array}{l}\text { Systolic BP } \\
(\mathrm{mm} \mathrm{Hg})\end{array}$ & $116.4 \pm 4.1$ & $115.0 \pm 3.5$ & $-1.4(-5.2-2.3)$ & $114.9 \pm 3.2$ & $117.5 \pm 4.1$ & $2.6(-1.4-6.7)$ & $115.7 \pm 3.8$ & $118.4 \pm 3.9$ & $2.7(0.3-5.1)$ & 14 \\
\hline $\begin{array}{l}\text { Diastolic BP } \\
(\mathrm{mm} \mathrm{Hg})\end{array}$ & $71.9 \pm 3.0$ & $70.2 \pm 2.7$ & $-1.7(-4.2-0.8)$ & $70.6 \pm 2.6$ & $70.2 \pm 2.6$ & $-0.4(-2.2-1.4)$ & $70.8 \pm 2.6$ & $72.0 \pm 2.7$ & $1.1(-1.9-4.2)$ & 14 \\
\hline $\begin{array}{l}\text { Heart rate } \\
\text { (bpm) }\end{array}$ & $58.5 \pm 2.3$ & $60.7 \pm 3.1$ & $2.2(-3.4-7.8)$ & $58.7 \pm 2.8$ & $59.1 \pm 2.6$ & $0.4(-3.0-3.8)$ & $60.1 \pm 1.8$ & $64.6 \pm 2.4^{*}$ & $4.4(0.3-8.5)$ & 14 \\
\hline $\mathrm{PWV}\left(\mathrm{m} \cdot \mathrm{s}^{-1}\right)$ & $6.0 \pm 0.2$ & $5.8 \pm 0.1$ & $-0.2(-0.5-0.2)$ & $5.9 \pm 0.1$ & $5.9 \pm 0.1$ & $-0.1(-0.3-0.2)$ & $5.8 \pm 0.1$ & $5.9 \pm 0.1$ & $0.1(-0.1-0.4)$ & 14 \\
\hline Alx@75 (\%) & $14.7 \pm 2.8$ & $14.0 \pm 3.2$ & $-0.7(-5.3-3.9)$ & $17.8 \pm 3.3$ & $13.9 \pm 3.4^{*}$ & $-3.9(-7.6--0.2)$ & $14.2 \pm 2.8$ & $12.7 \pm 2.5$ & $-1.5(-4.8-1.9)$ & 14 \\
\hline
\end{tabular}

$*=p<0.05$ vs. Pre-meal value; LCl=Lower $95 \%$ Confidence Interval, UCl=Upper $95 \%$ Confidence Interval 
explored this mechanism finding that a physiological dose of insulin resulted in decreased wave reflection in the aorta, but it took a pharmacological dose to produce an increase in forearm blood flow and a decrease in peripheral vascular resistance. This finding suggests that the decrease in wave reflection, hence Alx, is because of improved arterial compliance of arteries as opposed to changes in peripheral vascular resistance [33]. The reason for changes in Alx@75, independent of PWV supports the conclusion of McEniery et al. [37], that Alx@75 is the more sensitive marker of arterial compliance in younger individuals, and PWV is likely to be a better measure in older individuals.

This study also investigated the addition of oats to a high fat meal to determine their effects on arterial compliance; the reduction in Aix@75 following a high fat meal was abolished when oatmeal was consumed with a high fat meal. This is in agreement with the hypothesis that arise blood insulin increases vascular compliance, since a meal rich in oat $\beta$ glucan blunted the post-prandial insulin rise [38]. Thus the major finding in this study was the decrease in Alx@75 following a high-fat meal, an effect which was abolished by the inclusion of oats. These results suggest that there is a unique mechanism that enhances arterial compliance following the consumption of food, possibly linked to the vasodilatory effects of insulin. Alx@75 was also significantly related to BMI and WC. To conclude it may be that Alx@75 is a more sensitive marker of arterial compliance in a young healthy population as compared with PWV. Post-prandial changes in arterial compliance are known to be a risk-factor for cardiovascular disease, thus dietary components affecting this response (e.g. oats) may have an important influence in modulating the physiological post-prandial response following a high-fat meal, an area which requires further clarification. Future research should therefore continue to use FMD, PWV, and Alx@75 in parallel to further explore the reliability of these measurements as a marker of arterial compliance in comparison with FMD, to ascertain the true nature of the post-prandial endothelium-mediated vasodilatory response.

\section{Acknowledgement}

This study was funded by the Department of Dietetics, Nutrition, and Biological Sciences, Queen Margaret University. Grateful thanks are due to Ms Eipa Choudhury for her comments on the final version of the manuscript. 


\section{References}

1 De Caterina R, Zampolli A, Del Turco S, Madonna R, Massaro M, et al. (2006) Nutritional mechanisms that influence cardiovascular disease. Am J Clin Nutr 83: 421S-426S.

2 Stamatelopoulos K, Karatzi K, Sidossis LS (2009) Noninvasive methods for assessing early markers of atherosclerosis: the role of body composition and nutrition. Curr Opin Clin Nutr Metab Care 12: 467-473.

3 Kobayashi K, Akishita M, Yu W, Hashimoto M, Ohni M, et al. (2004) Interrelationship between non-invasive measurements of atherosclerosis: flow-mediated dilation of brachial artery, carotid intima-media thickness and pulse wave velocity. Atherosclerosis 173: 13-18.

4 Lane HA, Smith JC, Davies JS (2006) Noninvasive assessment of preclinical atherosclerosis. Vasc Health Risk Manag 2: 19-30.

5 Sutton-Tyrrell K, Newman A, Simonsick EM, Havlik R, Pahor M, et al. (2001) Aortic stiffness is associated with visceral adiposity in older adults enrolled in the study of health, aging, and body composition. Hypertension 38: 429-433.

6 Tomiyama H, Yamashina A (2010) Non-invasive vascular function tests: their pathophysiological background and clinical application. Circ J 74: 24-33.

7 Wilkinson IB, MacCallum H, Flint L, Cockcroft JR, Newby DE, et al. (2000) The influence of heart rate on augmentation index and central arterial pressure in humans. J Physiol 525 Pt 1: 263-270.

8 Wilkinson IB, Mohammad NH, Tyrrell S, Hall IR, Webb DJ, et al (2002) Heart rate dependency of pulse pressure amplification and arterial stiffness. Am J Hypertens 15: 24-30.

9 The Scottish Government (2013) The Scottish Health Survey.

10 Food Standards Agency (2007) FSA Nutrient and Food Based Guidelines for UK Institutions.

11 Vogel R A, Corretti MC, Plotnick GD (2000) The postprandial effect of components of the Mediterranean diet on endothelial function. J Am Coll Cardiol 36: 1455-1460.

12 Plotnick GD, Corretti MC, Vogel RA (1997) Effect of antioxidant vitamins on the transient impairment of endothelium-dependent brachial artery vasoactivity following a single high-fat meal. JAMA 278: $1682-1686$

13 Schinkovitz A, Dittrich P, Wascher TC (2001) Effects of a high-fat meal on resistance vessel reactivity and on indicators of oxidative stress in healthy volunteers. Clin Physiol 21: 404-410.

14 Clegg M, McClean C, Davison WG, Murphy HM, Trinick T, et al. (2007) Exercise and postprandial lipaemia: effects on peripheral vascular function, oxidative stress and gastrointestinal transit. Lipids Health Dis 6: 30.

15 Mc Clean CM, Mc Laughlin J, Burke G, Murphy MH, Trinick T, et al. (2007) The effect of acute aerobic exercise on pulse wave velocity and oxidative stress following postprandial hypertriglyceridemia in healthy men. Eur J Appl Physiol 100: 225-234.

16 Jakulj F, Zernicke K, Bacon SL, van Wielingen LE, Key BL, et al. (2007) A high-fat meal increases cardiovascular reactivity to psychological stress in healthy young adults. J Nutr 137: 935-939.

17 Djousse L, Ellison RC, McLennan CE, Cupples LA, Lipinska I, et al. (1999) Acute effects of a high-fat meal with and without red wine on endothelial function in healthy subjects. Am J Cardiol 84: 660-664.
18 Raitakari OT, Lai N, Griffiths K, McCredie R, Sullivan D, et al. (2000) Enhanced peripheral vasodilation in humans after a fatty meal. J Am Coll Cardiol 36: 417-422.

19 Sadiq Butt M, Tahir-Nadeem M, Khan MK, Shabir R, Butt MS, et al. (2008) Oat: unique among the cereals. Eur J Nutr 47: 68-79.

20 Davy BM, Davy KP, Ho RC, Beske SD, Davrath LR, et al. (2002) Highfiber oat cereal compared with wheat cereal consumption favorably alters LDL-cholesterol subclass and particle numbers in middle-aged and older men. Am J Clin Nutr 76: 351-358.

21 British Heart Foundation (2010) The Heart Health Roadshow.

22 Anderson JW, Hanna TJ (1999) Whole grains and protection against coronary heart disease: what are the active components and mechanisms? Am J Clin Nutr 70: 307-308.

23 Queenan KM, Stewart ML, Smith KN, Thomas W, Fulcher RG, et al. (2007) Concentrated oat beta-glucan, a fermentable fiber, lowers serum cholesterol in hypercholesterolemic adults in a randomized controlled trial. Nutr J 6: 6.

24 Katz DL, Nawaz H, Boukhalil J, Chan W, Ahmadi R, et al. (2001) Effects of oat and wheat cereals on endothelial responses. Prev Med 33: 476-484.

25 Katz DL, Evans MA, Chan W, Nawaz H, Comerford BP, et al. (2004) Oats, antioxidants and endothelial function in overweight, dyslipidemic adults. J Am Coll Nutr 23: 397-403.

26 Kerckhoffs DA, Hornstra G, Mensink RP (2003) Cholesterol-lowering effect of beta-glucan from oat bran in mildly hypercholesterolemic subjects may decrease when beta-glucan is incorporated into bread and cookies. Am J Clin Nutr 78: 221-227.

27 UK Government (2016) Measuring Blood Pressure - Top 10 Tips.

28 Smith L (2016) New AHA Recommendations for Blood Pressure Measurement.

29 Wildman RP, Mackey RH, Bostom A, Thompson T, Sutton-Tyrrell K, et al. (2003) Measures of obesity are associated with vascular stiffness in young and older adults. Hypertension 42: 468-473.

30 Berry SE, Tucker S, Banerji R, Jiang B, Chowienczyk PJ, et al. (2008) Impaired postprandial endothelial function depends on the type of fat consumed by healthy men. J Nutr 138: 1910-1914.

31 Vogel RA, Corretti MC, Plotnick GD (1997) Effect of a single high-fat meal on endothelial function in healthy subjects. Am J Cardiol 79: 350-354.

32 Laurent S, Cockcroft J, Van Bortel L, Boutouyrie P, Giannattasio C, et al. (2006) Expert consensus document on arterial stiffness: methodological issues and clinical applications. Eur Heart J 27: 2588-2605.

33 Westerbacka J, Wilkinson I, Cockcroft J, Utriainen T, Vehkavaara $\mathrm{S}$, et al. (1999) Diminished wave reflection in the aorta. A novel physiological action of insulin on large blood vessels. Hypertension 33: 1118-1122.

34 Scherrer U, Randin D, Vollenweider P, Vollenweider L, Nicod P, et al. (1994) Nitric oxide release accounts for insulin's vascular effects in humans. J Clin Invest 94: 2511-2515.

35 Harris RA, Tedjasaputra V, Zhao J, Richardson RS (2012) Premenopausal women exhibit an inherent protection of endothelial function following a high-fat meal. Reprod Sci 19: 221-228.

36 Fitch RM, Vergona R, Sullivan ME, Wang YX (2001) Nitric oxide synthase inhibition increases aortic stiffness measured by pulse wave velocity in rats. Cardiovasc Res 51: 351-358. 
37 McEniery CM, Wallace S, Mackenzie IS, McDonnell B, Yasmin, et al. (2006) Endothelial function is associated with pulse pressure, pulse wave velocity, and augmentation index in healthy humans. Hypertension 48: 602-608.
38 Juvonen $K R$, Salmenkallio-Marttila $M$, Lyly $M$, Liukkonen $K H$, Lahteenmaki L, et al. (2011) Semisolid meal enriched in oat bran decreases plasma glucose and insulin levels, but does not change gastrointestinal peptide responses or short-term appetite in healthy subjects. Nutr Metab Cardiovasc Dis 21: 748-756. 